\title{
Secondary User Access for IoT Applications in the FM Radio Band using FS-FBMC
}

\author{
Kenneth W. Barlee, Robert W. Stewart, Louise H. Crockett \\ Software Defined Radio Research Laboratory, Centre for Whitespace Communications \\ Dept. of Electronic and Electrical Engineering, University of Strathclyde, Glasgow, G1 1XW, Scotland \\ kenneth.barlee@strath.ac.uk
}

\begin{abstract}
In this paper a Dynamic Spectrum Access (DSA) Physical layer (PHY) technique is proposed that allows Secondary User (SU) access to the traditional FM Radio spectrum (88-108 MHz) for alternative data communication applications. FM radio waves have excellent propagation characteristics for long distance transmission, and have high levels of penetration through buildings. Using tools such as a structured geolocation database of licensed Primary User (PU) FM Radio transmitters, unlicensed SUs can access portions of the $20 \mathrm{MHz}$-wide band and transmit signals that place spectral 'holes' with suitable guard bands around all known PUs. Based on the PU protection ratios published by Ofcom and the FCC, the operation of a FBMC (Filter Bank Multi-Carrier) transmitter is demonstrated for an urban environment, and through 'field test' simulation it is shown that the Out Of Band (OOB) leakage of the proposed PHY (energy in the 'holes' that can interfere with the $P U$ ) is $47 \mathrm{~dB}$ lower than that of using an equivalent OFDM PHY. The results show that the proposed PHY is a suitable candidate for DSA-SU communication (e.g. in smart city IoT applications), whilst ensuring the integrity of incumbent PU signals.
\end{abstract}

\section{INTRODUCTION}

Over recent years, the proliferation of wireless mobile services and applications has led to the so called 'spectrum crunch', whereby existing allocated radio spectrum is often presented as being nearly at capacity. Yet upon inspection, large swathes of the spectrum appear to be underutilized or not in use at all. This spectrum shortage 'paradox' [1] is well understood to be a result of the largely static allocation policy used by regulators around the world, rather than the physical shortage of available bands. To give an example, in the Highlands and Islands of Scotland where cellular service is sparse and often limited only to $2 \mathrm{G}$, there is no shortage of unused 'cellular' bands. Fixed allocation policies mean that there are many unused (but PU allocated) bands, such as those for FM Radio, Digital TV and cellular services all across the country. With no structured models in place to allow the sharing of these bands, they simply lie empty. Using new spectrum access techniques however, they could be targeted for 5G and IoT applications.

Dynamic Spectrum Access (DSA) is a method of spectrum sharing whereby Secondary User (SU) nodes and networks are able to access the spectrum and establish communication channels on an unlicensed (but controlled) basis. With appropriate regulatory frameworks, DSA will see licensed Primary Users (PUs) encouraged to share bands they are not using in order to allow the spectrum to be used more efficiently. Ideally SUs would be spectrally aware, and capable of identifying bands they can access without causing unacceptable interference to incumbent PUs [2]. After identifying suitable bands, the SUs can create communications channels in them using multicarrier modulation schemes. As the bands are likely to be NonContiguous (NC), dynamic modulation techniques with variable spectral masks should be used. These requirements point to Software Defined Radio (SDR) implementations. Here, engineers can develop flexible Physical Layers (PHYs) for implementation on programmable hardware. To date, regulators such as the FCC (USA) and Ofcom (UK) have not permitted this type of DSA. Instead, geolocation databases have been developed to provide information (on request) about which bands are available at any particular location, and the transmit power permitted in each of them. With the current rules, SUs can only choose channels from this list.

Recent examples of DSA in action include the many TV White Space (TVWS) pilot networks implemented around the globe. The Centre for White Space Communications has been involved in the design and operation of three pilot networks in Scotland: on the Isle of Bute in 2011, Glasgow city centre in 2014 [3], and most recently in the remote Orkney Islands in 2016 [4]. With funding from the Scottish Government, the Orkney project has seen DSA-enabled fixed Internet access provided to rural communities, and 'nomadic' access on inter-island ferries.

In this paper a Filter Bank Multi-Carrier (FBMC) transmitter is introduced that can be used in combination with a database (or local knowledge of PU broadcasts) to allow SU access in the FM Radio band. Methods are presented that enable the proposed SU radio to coexist with FM broadcasts without any discernible interference to the PU signals.

\section{FM RADIo BAND DyNAMIC SPECTRUM ACCESS}

While the recent focus of research, industrial, and regulatory bodies has been on implementing TVWS in the UHF band (470$890 \mathrm{MHz}$ ), the techniques they have developed are also applicable to other bands. FM Radio waves (88-108 MHz in most countries) have excellent propagation characteristics, and are able to diffract around objects such as hills and human-made structures, and penetrate through buildings well. Although 2017

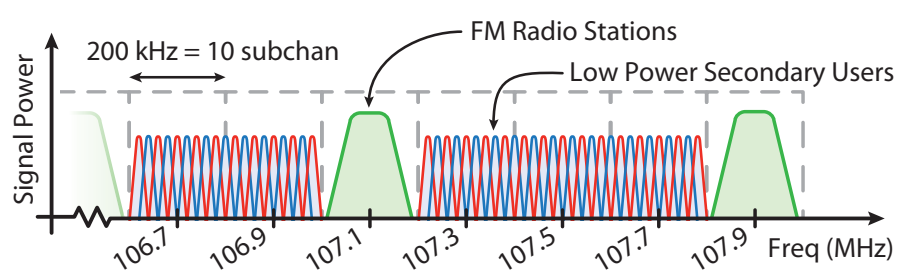

Fig. 1. SUs broadcasting in available channels of the FM Radio Band. 
saw Norway ending FM Radio transmission in favour of a new digital solution, in many nations the reliance on, and investment in 'trusty' FM Radio will likely mean that its existence is assured for many years to come.

A recent study conducted in the USA has shown that a significant portion of the 100 individual $200 \mathrm{kHz}$-wide FM Radio channels are in fact unallocated at any given location [5]. The average findings across the country suggest that, in regions with populations between 100,000 and 1 million, only around $25 \%$ of the channels are in use on average [6]. In rural areas, the figures are much lower. Of course this is not to say that there will always be $15 \mathrm{MHz}$ of the FM band unallocated in these areas; and further, when FCC rules on minimum distance separations (protected radii around transmitters; essentially frequency guard bands) are taken into account, the amount of 'vacant' spectrum is actually lower. However, the findings in [6] do provide a strong argument that the FM band is a suitable candidate for enabling SU access.

In this paper a SU radio is proposed that uses an FBMC scheme with frequency overlapping subchannels spaced $20 \mathrm{kHz}$ apart $(20 \mathrm{kHz}=1 / 10$ th of a standard 'FM channel'). This means 10 subchannels fit inside an 'FM channel', as illustrated in Fig. 1. It is an essential requirement that subchannels at frequencies known to be 'in use' by the PU can be disabled and spectrally 'knocked-out', in order to ensure that the PU signal is protected. The set of subchannels to disable would be determined either by consulting a (new) FM Radio geolocation database, or locally by the SU, using a regulator-approved spectral sensing technique.

In order to ensure the protection of PUs, the Adjacent Channel Leakage Ratio (ACLR) of the modulation scheme in use by the SU must be low (i.e. disabled subchannels must have minimal Out Of Band (OOB) leakage power levels to prevent interference with the PU). NC- OFDM (one of the favorite NC schemes presented in the literature-[7][8]) allows subcarriers to be disabled, but due to the fact it uses rectangular pulse shaping, it has very high OOB power levels [9]. FBMC can also transmit NC channels, and depending on the prototype filter chosen, can achieve excellent ACLR levels [10]. (The prototype filter's frequency response is used as the basis of all filters in the bank).

As DSA is currently not implemented in the FM Radio band, no ACLR limits have been set by regulators; but inspiration can be taken from the FCC and Ofcom TVWS limits and the FM Radio regulations. The FCC has set the maximum OOB power $55 \mathrm{~dB}$ lower than in-band levels [11], Ofcom requires a maximum leakage of $-46 \mathrm{dBm}$ ( $82 \mathrm{~dB}$ at maximum transmit power) [12], and the ITU-R has set the co-channel interference limit for FM Radio to $45 \mathrm{~dB}$ [13].

Following a review of FBMC in Sec. III, Sec. IV of this paper discusses the design of the SU transmitter PHY for the FM Radio band. The radio is developed in Simulink ${ }^{\circledR}$, and simulations are performed to analyze the interference it presents to PU signals under different transmit power levels. A test methodology is discussed in Sec. V, and qualitative and quantitative results are obtained and presented. The proposed FBMC transmitter is shown to cause significantly less interference than an equivalent NC-OFDM implementation. A discussion of potential applications is given in Sec. VI, and conclusions are drawn in Sec. VII.

\section{REVIEW OF FBMC/OQAM SYSTEMS}

In FBMC, a communication channel is split up into a number of subchannels by a bank of filters. If the passbands of filters are spectrally separated, different modulation schemes can be used simultaneously across the set of subchannels [14]. Frequency overlapping FBMC is more spectrally efficient, but requires the use of an orthogonal modulation technique called Offset QAM (OQAM) [15]. OQAM orthogonality is achieved by inputting the real and imaginary components of complex QAM symbols individually to the Inverse Fast Fourier Transform (IFFT), and transmitting them with a time offset of half a symbol [16]. In a practical sense, this means doubling the symbol rate and alternately inputting the real and imaginary components to evenindexed and odd-indexed subchannels (IFFT bins) [17]. Splitting the QAM symbols in this way allows for maximum spectral efficiency to be reached.

One of the benefits that FBMC/OQAM systems have over OFDM/QAM is that they do not require a Cyclic Prefix (CP) in order to protect the orthogonality of the subcarriers. The real prototype filter used for FBMC/OQAM results in excellent frequency and time localisation, meaning that it is less susceptible to Inter-Carrier and Inter-Symbol Interference (ICI and ISI) [18]. Another advantage becomes clear in the Multiple Access (MA) scenario. Here, groups of subchannels are aggregated together, and allocated to different users (as in LTE). With OFDM/MA, it is critical that all of the users' signals are received at the same time and at the correct frequencies in order to maintain orthogonality conditions in the receive FFT. In contrast, once an empty OQAM subchannel is left between groups, transmitter synchronisation between MA nodes is not required when transmitting to an FBMC/OQAM basestation [17]. This is an important point, since it can drastically simplify the architecture of transmit-only radio nodes; for example IoT nodes that only broadcast sensor readings.

Two of the more common architectures of FBMC that feature in the literature are the Polyphase Network (PPN-FBMC) and Frequency Spreading (FS-FBMC). Configured with equivalent parameters and prototype filter designs, both of these architectures will achieve the same spectra [19]; and, as will be discussed, there are advantages and disadvantages to each of them. The filter bank exists either in the time- or frequencydomain, depending upon which architecture is chosen.

In the PPN-FBMC/OQAM transmitter, $M$ modulated OQAM symbols ( $M / 2$ complex QAM symbols) are input to an IFFT, and the output is passed to a time-domain polyphase filter bank network. The bank's prototype filter is designed based on an overlapping factor, $K$ [20]. The advantage of this design is that the IFFT size is limited to $M$ (rather than $M K$, as with FSFBMC). There are drawbacks, however, with the PPN- receiver. Equalisation must be carried out as a complex multi-tap process in the time-domain (unlike the single tap process required for OFDM), which adds a processing overhead and a time delay [14]. Carrier Frequency Offset (CFO) and Timing synchronisation must also be performed in the time-domain, prior to the receiver's FFT. Time-domain misalignment significantly reduces the amount of channel delay spread the receiver can compensate for; hence this architecture does not perform well in multipath 


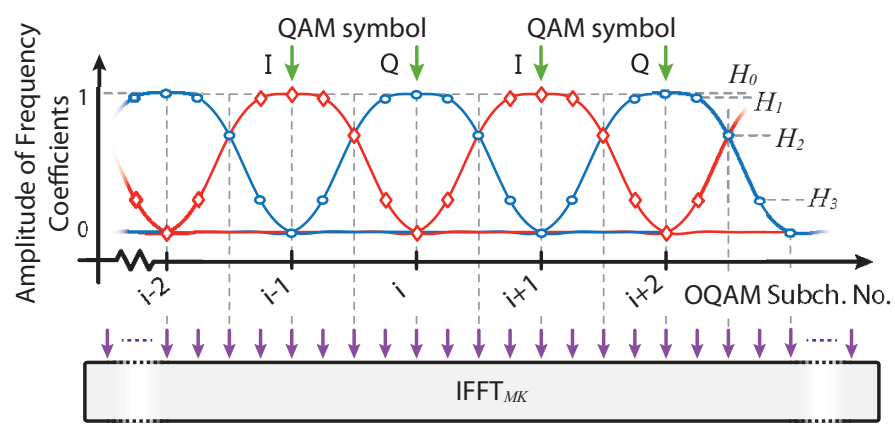

Fig. 2. Part of a FS-FBMC/OQAM transmitter with $K=4$ PHYDYAS filter, highlighting the subchannel overlap.

environments [10].

In comparison, the FS-FBMC/OQAM transmitter sees $M$ modulated OQAM symbols upsampled by $K$ in the frequencydomain ('frequency spread') and convolved with the frequency coefficients of the prototype filter. Next, the $M K$ filtered samples are passed into the IFFT, and $2 K$ of the time-domain outputs are 'overlapped and summed' together [17]. While there is a significant amount of redundant processing in this transmitter, the gains are made in the FS- receiver. CFO and Timing synchronisation systems can be positioned after the receiver's FFT, and similarly, equalisation can be performed as a single tap operation in the frequency-domain (in the same way as with OFDM) [14]. This means that it is better able to compensate for the effects of multipath. Additionally, the FFT operation can be carried out asynchronously (i.e. without prior knowledge of the time domain alignment) [21]. Another benefit to this receiver architecture is that the higher frequency domain resolution of the FFT ( $M K$ rather than $M$ bins) will lead to more accurate sensing results, should spectrum sensing be being carried out in the radio.

Prototype filters are normally designed in the frequencydomain, regardless of which architecture is latterly used. The filter is symmetric, and the number of non-zero coefficients of the filter is given by $P=2 K-1$ [14]. The higher the $K$ value, the better the ACLR; but a trade-off is made to keep $K$ small. This has led to $K=4$ becoming a common value, as used in the EU FP7 PHYDYAS project [17]. The coefficients are calculated such that (1) is satisfied:

$$
\frac{1}{K} \sum_{k=-K+1}^{K-1}\left|H_{k}\right|=1 .
$$

With $K=4$, this gives frequency coefficients of

$$
\begin{array}{ll}
H_{0}=1 & H_{1}=H_{-1}=0.97196 \\
H_{2}=H_{-2}=\sqrt{2} / 2 & H_{3}=H_{-3}=0.235147,
\end{array}
$$

resulting in the filterbank shown in Fig. 2. It is key to note here that only subchannels that are direct neighbours overlap.
Disabling a single subchannel (which can be acheived by simply inputting a zero-valued OQAM symbol to the transmitter) creates spectral separation between the remaining subchannels, removing the requirement of orthogonality between them. Disabling a number of neighbouring subchannels will create a 'hole' in the generated FBMC/OQAM signal (essentially making it NC). The roll-off of the $K=4$ filter means that (within 3 subchannel spacings) the power of the OOB leakage in the holes will be $80 \mathrm{~dB}$ lower than the in-band level [22]. Unlike NCOFDM, this signal can be moulded to fit around existing PUs, and broadcast with minimal interference to them.

While PPN-FBMC is the de facto architecture because of its efficient implementation, the synchronisation benefits of the FSFBMC receiver mean that the frequency spreading technique is the architecture of choice when designing radios for use in a DSA environment [10]. Because the designs are equivalent, in theory it is possible to have a mixed architecture radio; i.e. using a PPNFBMC transmitter and an FS-FBMC receiver [19]. This is an attractive concept, as it results in the 'best of both worlds'. For the purposes of this initial investigation however, the FS-FBMC architecture was chosen.

\section{DESIGN OF AN FS-FBMC TRANSMITTER FOR THE FM RADIO BAND}

A system sampling rate $\left(f_{s}\right)$ of $20.48 \mathrm{MHz}$ was chosen. With $M=1024$, the (overlapping) OQAM subchannels have a bandwidth of $40 \mathrm{kHz}$, and a total of 10 OQAM subchannels can fit inside each standard $200 \mathrm{kHz}$ wide 'FM channel'. Given the ACLR limits discussed in Sec. II, it was decided that the PHYDYAS filter with its $80 \mathrm{~dB}$ OOB attenuation would be sufficient for the exercise.

The transmitter was developed in Simulink, using low level components from the MathWorks HDL Coder library. This means that, later, the design can be targeted to programmable SDR hardware. An equivalent system was also built for an NCOFDM transmitter, to allow for comparison.

The design sees QAM symbols generated at a rate of $T=1 / f_{s}$. A channel mask is applied, which will see some subchannels disabled to protect the PUs (this will be discussed in greater detail in Sec. V). The symbols undergo OQAM preprocessing, are upsampled by a factor of $K$, and convolved with the frequency domain PHYDYAS filter coefficients. Note that these operations increase the sampling rate to $2 K f_{s}$. The samples pass through an IFFT to generate time domain FBMC symbols, and these are fed into a bank of FIFOs (First-In First-Out memory units). The outputs are clocked such that samples are output at the original symbol rate, $T$, and the FBMC symbols are 'overlapped' and summed together in the time domain. The architecture of the transmitter is illustrated in Fig. 3.

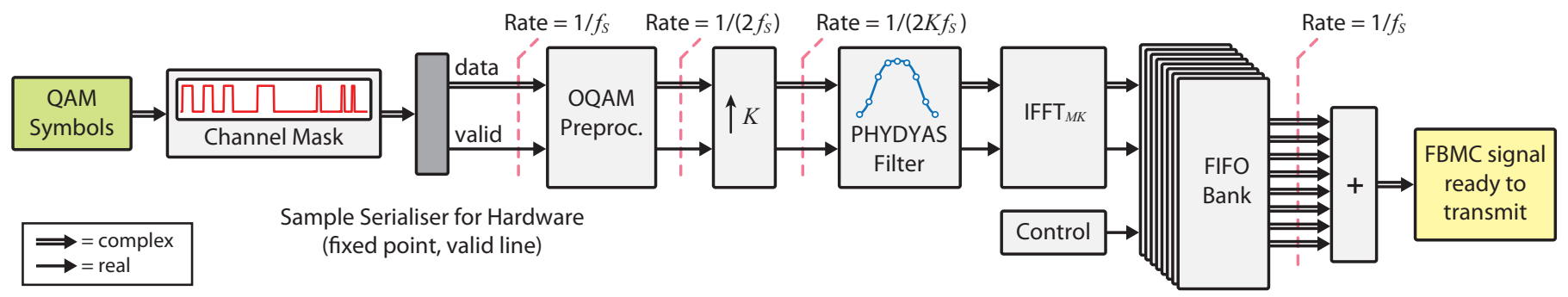

Fig. 3. Block diagram of the SDR hardware synthesize-able FS-FBMC Transmitter. 


\section{TESTING SU INTERFERENCE LEVELS}

It is not currently legal to broadcast unlicensed SU signals in the FM Radio band, so in order to test the concept, 'field test' simulations were performed instead to explore SU interference levels (i.e. the level of interference caused by the SU to the PU). Suitable Radio Frequency (RF) recordings of the FM Radio band had to be obtained to do this, and these were acquired with a USRP B210 SDR from the top of a building on the Strathclyde University campus $\left(55.860876^{\circ} \mathrm{N}, 4.243672^{\circ} \mathrm{W}\right)$. An 8 second recording of the entire band was made, that would later allow interference to be gauged both quantitatively and qualitatively (via listening tests). A total of 22 receivable (demodulate-able!) FM stations were found. Most of these were transmitted from Blackhill in central Scotland $\left(55.861944^{\circ} \mathrm{N}, 3.872500^{\circ} \mathrm{W}\right), 23 \mathrm{~km}$ away. There were 7 local area stations, and also 6 duplicates from the Darvel transmitter in Ayrshire $\left(55.579430^{\circ} \mathrm{N}, 4.289775^{\circ} \mathrm{W}\right)$, $31 \mathrm{~km}$ away. The duplicate Darvel stations were much lower power, but they are not intended to service the Glasgow area; albeit their existence means they were treated as PUs.

The USRP B210 used for the recording was un-calibrated, which means that the samples received by the computer all had relative power levels, and hence appropriate scaling was required in order to make fair comparisons later. Technical parameters regarding the UK's FM transmitters were downloaded from the Ofcom website [23], and calculations were performed to estimate the average expected receive power of the $\mathrm{BBC}$ national stations transmitted from the Blackhill transmitter. Using the Friis free space channel model, the expected power was found to be 30 $\mu \mathrm{W} /-15.3 \mathrm{dBm}$. As this model does not take into account the antenna height difference (which was approximately $400 \mathrm{~m}$ between the transmitter and the USRP receiver), a second calculation was performed using the Perez-Vega Zamanillo / FCC $\mathrm{F}(50,50)$ free space VHF model for comparison. A MATLAB implementation was created based on [24], and this showed the expected receive power to be $24 \mu \mathrm{W} /-16.2 \mathrm{dBm}$. While the $\mathrm{F}(50,50)$ model is likely more accurate, it was noted that neither it nor the Friis model take into account the geography of the transmit channel, so some further attenuation should be expected. In light of this, the power of the recorded FM signal was adjusted such that the average Blackhill BBC station power was around $-16.5 \mathrm{dBm}$.

\section{A. Dynamic Generation of a Spectral Mask}

With no existing geolocation database for the FM frequency band, a SU channel mask was not readily available for the recording location; therefore a mask was created based on the FM
Radio stations detected in the recording as well as those listed in the Ofcom documents. The centre frequencies of the FM stations were noted, and a MATLAB ${ }^{\circledR}$ script was written to automate the process. An arbitrary guard band size of $300 \mathrm{kHz}$ (1.5 FM Radio channels/ 15 OQAM subchannels) was chosen, meaning a total of 40 OQAM subchannels would be disabled around each active FM Radio signal. (The optimum guard band size is a subject of further research). Running the script, a mask was generated with 749 of the 1024 OQAM subchannels disabled, leaving $275(26 \%)$ active. This is the mask shown in Fig. 3. The mask was loaded into the transmitter model, and an FBMC signal with spectral 'holes' was produced. Additionally, an NC-OFDM signal with the same number of subcarriers and the same channel mask was also created, to allow for comparison.

\section{B. Configuring the SU Transmit Power Level}

As there are no existing regulations for SUs transmitting in the FM Radio band, there are no rules for transmit power levels. Therefore, to set a fair and reasonable power level, it was noted that in the EU, the maximum WiFi transmit power is $0.1 \mathrm{~W} / 20$ $\mathrm{dBm}$. The FCC has a higher limit of $4 \mathrm{~W} / 36 \mathrm{dBm}$ for the USA, which is the same power agreed upon for TVWS broadcasts by the FCC and Ofcom [25]. The interference levels produced with SU transmit powers in this range were therefore explored.

The FBMC and NC-OFDM SU signals were configured with the various power levels, and added to the power-adjusted recording of the FM band. This resulted in spectra similar to the one shown in Fig. 4. In this particular case, the power of the SU has been set to $0.1 \mathrm{~W}$. It is evident from the plot (even without the need for further calculation) that, at this low transmit power, the NC-OFDM signal's OOB leakage will interfere with many of the PU FM channels. However, the PHYDYAS FBMC signal fits well around them, having an OOB leakage level below the noise floor of the FM band in this case.

In order to explore the interference levels, each of the active FM Radio channels (from the $F M+S U$ overlay signals) had to be isolated. The $F M+S U$ overlay signals were mixed with a complex sine wave to shift each active FM channel to baseband in turn, and decimation by a factor of 100 was performed.

\section{Quantitative Measurement of Interference}

The average power for each of the FM and SU signals $\left(P_{F M}\right.$ and $\left.P_{S U}\right)$ in active FM Radio channels under various transmit power levels were found using (2) and (3), respectively.

$$
P_{F M}=\frac{1}{N} \sum_{n=1}^{N}\left|s_{F M}(n)\right|^{2}
$$

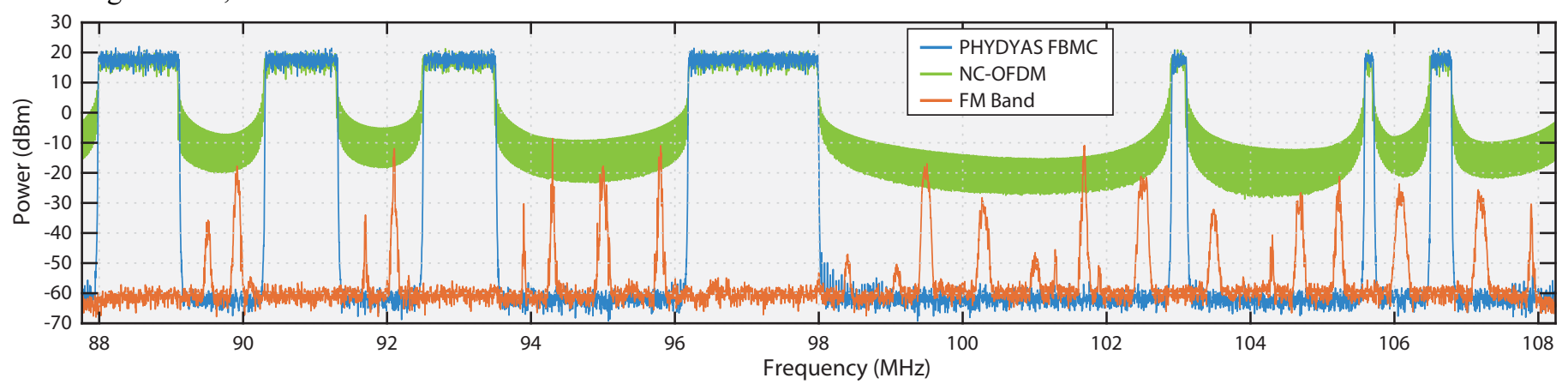

Fig. 4. Spectrum Analyzer window showing the PHYDYAS FBMC, NC-OFDM and recorded FM radio signals $-S U$ transmit power $=0.1 \mathrm{~W} / 20 \mathrm{dBm}$. 


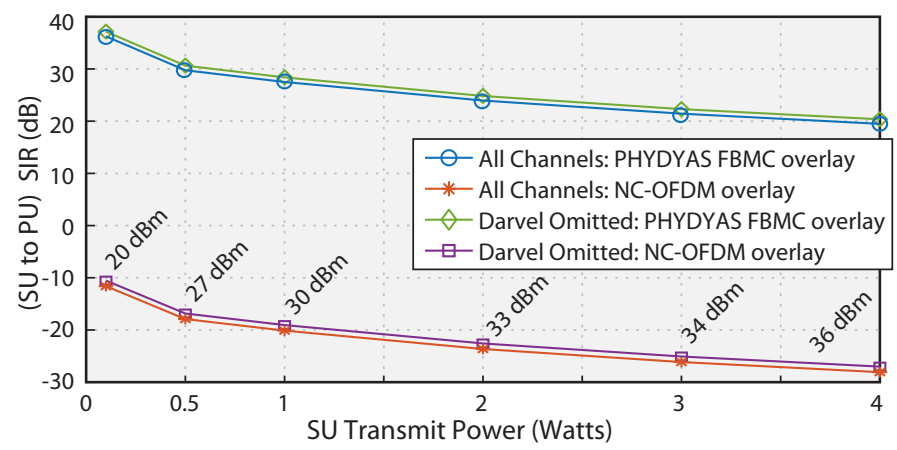

Fig. 5. Plot of average SIR (SU to PU) for various SU transmit powers.

$$
P_{S U}=\frac{1}{N} \sum_{n=1}^{N}\left|s_{F M+S U}(n)\right|^{2}-P_{F M},
$$

(where $s_{F M}$ is the power-adjusted FM signal and $s_{F M+S U}$ is the $F M+S U$ overlay), allowing the Signal to Interference Ratio (SIR) to be calculated (in $\mathrm{dB})$ :

$$
S I R_{d B}=10 \log _{10}\left(\frac{P_{F M}}{P_{S U}}\right) .
$$

Fig. 5 shows the average SIR experienced across all FM Radio channels, against SU transmit power. Additionally, the results omitting the signals transmitted from Darvel (which are probably fair to consider as non-PU) are shown. Here the SIR of the weak duplicate channels is emitted from the calculations.

This plot shows that overlaying the PHYDYAS FBMC signal on the FM spectrum results in $\sim 47 \mathrm{~dB}$ improvement in PU SIR when compared with overlaying the NC-OFDM signal. With an SU transmit power of $0.1 \mathrm{~W}$ (as demonstrated in Fig. 4), the interference of the PHYDYAS signal is negligible, however NCOFDM is shown to significantly degrade the quality of the FM signal. The NC-OFDM case worsens as the transmit power is increased, resulting in such serious interference that, at $4 \mathrm{~W}$, the power of the NC-OFDM OOB leakage is on average 625 times $(28 \mathrm{~dB})$ greater than that of the FM Radio signal. In comparison, the leakage power of the PHYDYAS SU is 88 times $(19 \mathrm{~dB})$ lower than the power of FM signal. From a numerical point of view, the calculated SIR values show that there is a significant benefit to using PHYDYAS instead of NC-OFDM for NC SU access.

\section{Qualitative Measurement of Audio Interference}

Each of the 22 active FM Radio channels from the poweradjusted recording of the FM band were processed by a Complex Frequency Discriminator in Simulink, in order to extract the audio signal contained within [26]. The outputs were saved to .wav files, creating a 'golden' reference set of demodulated FM audio signals for later comparison. Next, the $F M+S U$ overlay signals for each of the different SU transmit powers were demodulated, so that the audio quality of each FM channel could be rated in the presence of the SU signals.

The simple Mean Opinion Score (MOS) method was adopted for qualitative listening comparison, which saw a score between 1-5 awarded by 8 listeners depending on perceived quality ( $1=$ terrible, $5=$ excellent). The results of this exercise are shown in Fig. 6. While these are inherently subjective, the trend is consistent with the quantitative results obtained. As the NCOFDM transmit power is increased, the quality of the received

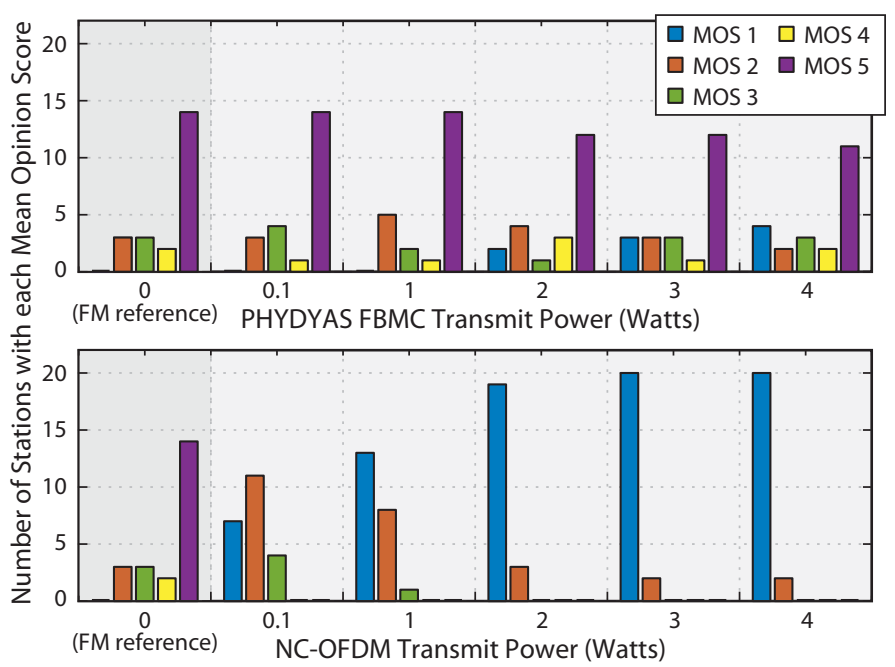

Fig. 6. Plots of station audio quality MOS for PHYDYAS FBMC and NC-OFDM overlay signals.

PU signals rapidly decreases; whereas with the PHYDYAS FBMC signal, the quality remains high. At the maximum transmit power tested, 11 stations (of the 14 originally rated at MOS-5) could still be received perfectly, without any noticeable interference. The 4 stations that did become overpowered by PHYDAS OOB interference at $4 \mathrm{~W}$ transmit power were all duplicate stations from the Darvel transmitter. All of the demodulated signals have been catalogued to allow the reader to make their own judgements of audio quality. They are available for download in reference [27].

\section{PHySiCAL LAYER PROTOCOL}

If transmitted FBMC bursts were of infinite length, and no synchronisation overhead was required, the bandwidth efficiency of the FBMC transmitter would be 100\% [9]. However, bursts would have a finite duration, meaning filter transitions need to be accounted for; and preambles and scattered pilots would likely be required in order to keep a corresponding receiver synchronised [28]. This is demonstrated in Fig. 7. The design and length of the preamble, the overall burst size, and the number of pilots used will ultimately depend on the intended application of the radio. Further work will be required to identify the most suitable parameters.

With the channel mask used in this example, 275 of the 1024 channels are available for use. If the radio were to be deployed in a point-to-point link, a total $\mathrm{SU}$ channel bandwidth of $5.5 \mathrm{MHz}$ would be available. In the MA scenario, some additional subchannels would need to be disabled in order to create the required spectral separation between those aggregated. To give an example with the mask in question, if groups of 4 OQAM subchannels +1 separator were used, 55 aggregated channels would be available (from a single FBMC basestation), each with a bandwidth of $80 \mathrm{kHz}$.

MA also opens the doors to the possibilities of reduced

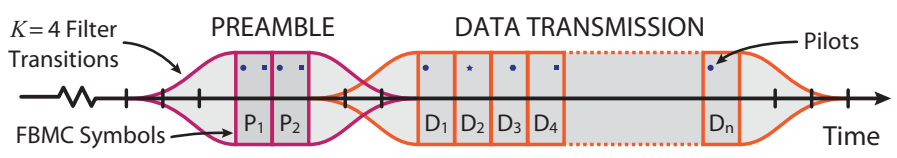

Fig. 7. Potential burst structure for the PHYDYAS FBMC scheme [20] 
complexity transmitters. Only 16 IFFT bins (4 OQAM subchannels $\times K$ ) would be required for transmitters in this MA example, drastically decreasing the number of multiply operations required during every sample period (a saving of 49088 multiplies in the Radix 2 IFFT alone); and reducing the rate at which samples have to be processed. Ultimately, this means transmitters can be implemented with lower cost hardware.

\section{CONCLUSIONS}

In this paper a novel PHY strategy for SU access to the FM Radio spectrum has been presented. Current FM Radio usage in homes, cars, and portable devices, alongside the slow uptake of new digital alternatives, means that FM Radio is expected to be around for many years to come. Not all FM channels are in use, so there is an opportunity to employ DSA methods to share the spectrum. DSA radios must be flexible and efficient in their use of available NC channels while protecting incumbent PU signals; and the designed PHYDYAS FBMC/OQAM transmitter has demonstrated these capabilities. Through 'field test' simulations it has been shown to provide a $47 \mathrm{~dB}$ improvement in PU interference levels over an equivalent NC-OFDM architecture. DSA techniques such as this could bring a new lease of life to the old FM spectrum.

Ongoing investigations at the University of Strathclyde are exploring the design of very low bit rate FBMC systems for IoT in the FM band, prototyped using targeted SDR hardware. Data rates, geographical coverage and performance are being demonstrated and assessed. Going forward, it is proposed that DSA FBMC systems, such as the one presented in this paper, (underpinned by either a geolocation database or spectrum sensing to identify suitable channels) are prime PHY candidates for the new $5 \mathrm{G}$ IoT era.

Acknowledgements: This work was co-funded by the MathWorks DCRG grant on Dynamic Spectrum Access for $5 G$ Communications - 2016-2018.

\section{REFERENCES}

[1] Q. Zhao and B. M. Sadler, "A Survey of Dynamic Spectrum Access", in IEEE Signal Process. Mag., vol. 24 no. 3, pp. 79-89, May 2007.

[2] E. Hossain, D. Niyato, Z. Han, Dynamic Spectrum Access and Management in Cognitive Radio Networks, 1st Edition, Cambridge, UK: Cambridge University Press, 2009.

[3] D. Crawford. (2016, May). The Glasgow TVWS Pilot, Introduction and Overview. CWSC. [Online]. Available: http://www.wirelesswhitespace.org/rdr/glasgowpilot.html.

[4] G. Whitton. (2015, May). TVWS Orkney Islands. CloudNet ITSolutions Ltd. [Online]. Available: http://www.wirelesswhitespace.org/rdr/orkneypilot.html.

[5] D. Otermat, C. Otero, I. Kostanic, "Analysis of the FM Radio Spectrum for Internet of Things Opportunistic Access Via Cognitive Radio", in WF-IoT'15, Milan, IT, pp. 166-171, Dec 2015.

[6] D. Otermat, C. Otero, I. Kostanic, "Analysis of the FM Radio Spectrum for Secondary Licensing of Low-Power Short-Range Cognitive Internet of Things Devices", in IEEE Access, Oct 2016.

[7] R. Rajbanshi, A. Wyglinski, G. Minden, “An Efficient Implementation of NC-OFDM Transceivers for Cognitive Radios”, in CROWNCOM'06, Mykonos, GR, pp. 1-5, Jun 2006.
[8] B. Farhang-Boroujeny, R. Kempter, "Multicarrier communication techniques for spectrum sensing and communication in cognitive radio", in IEEE Commun. Mag., vol. 46 no. 4, pp. 80-85, Apr 2008.

[9] R. Gerzaguet et al., "The 5G candidate waveform race: a comparison of complexity and performance", in EURASIP Journal on Wireless Commun. and Networking, Jan 2017.

[10] V. Berg, J. Doré, D. Noguet, “A Flexible FS-FBMC Receiver for Dynamic Spectrum Access", in CROWNCOM'14, Oulu, FI, pp. 285-290, Jul 2014.

[11] FCC, "Unlicensed Operation in the TV Broadcast Bands", US Federal Register, vol. 74 no. 30, pp. 7314-7332, Feb 2009.

[12] Ofcom, "Cognitive Access, Statement on licence-exempting cognitive devices using interleaved spectrum", Jul 2009.

[13] ITU-R, "Planning standards for terrestrial FM sound broadcasting at VHF", Recommendation BS.412-9, Dec 1998.

[14] M. Bellanger, "FS-FBMC: a flexible robust scheme for efficient multicarrier broadband wireless access", in IEEE Globecom Workshops, Anaheim, USA, Dec 2012.

[15] P. Siohan, "Analysis and Design of OFDM/OQAM Systems Based on Filterbank Theory", in IEEE Trans. On Signal Proc., vol. 50 no. 5, pp. 1170-1183, May 2002.

[16] B. Farhang-Boroujeny, "OFDM Versus Filter Bank Multicarrier", in IEEE Signal Process. Mag., vol. 28 no. 3, pp. 92-112, May 2011.

[17] M. Bellanger. (2010, Jun). FBMC physical layer: a primer, PHYDYAS. [Online]. Available: http://www.ict-phydyas.org/ teamspace/internal-folder/FBMC-Primer_06-2010.pdf.

[18] M. Schellmann et al., "FBMC-based air interface for 5G Mobile: Challenges and proposed solutions", in $C R O W N C O M^{\prime} 14$, Oulu, FI, pp. 102-107, Jul 2014.

[19] D. Mattera, M. Tanda, M. Bellanger, "Analysis of an FBMC/ OQAM scheme for asynchronous access in wireless communications", in EURASIP Journal on Advances in Signal Processing, Mar 2015.

[20] A. Viholainen, T. Ihalainen, T. Stitz, M. Renfors, M. Bellanger, "Prototype filter design for filter bank based multicarrier transmission", in EUSIPCO'09, Glasgow, UK, pp. 1359-1363, Aug 2009.

[21] J. Doré, V. Berg, N. Cassiau, D. Kténas, "FBMC receiver for multi-user asynchronous transmission on fragmented spectrum", in EURASIP Journal on Advances in Signal Processing, Mar 2014.

[22] A. Viholainen, M. Bellanger, M. Huchard. (2009, Jan). PHYDYAS D5.1. [Online]. Available: http://www.ict-phydyas.org/ delivrables/PHYDYAS-D5-1.pdf.

[23] Ofcom. (2017, Aug). Technical parameters for broadcast radio transmitters. [Online]. Available: https://www.ofcom.org.uk/ spectrum/information/radio-tech-parameters.

[24] C. Pérez-Vega and J. Zamanillo. (2002, Jun). Path-Loss Model for Broadcasting Applications and Outdoor Communication Systems in the VHF and UHF Bands. [Online]. Available: http://personales.unican.es/perezvr/pdf/FCC\%20Model02.PDF.

[25] Ofcom, "Implementing TV White Spaces", Feb 2015.

[26] R. Stewart, K. Barlee, D. Atkinson, L. Crockett, Software Defined Radio using the MATLAB \& Simulink and the RTL-SDR. Glasgow, UK: Strathclyde Academic Media, Sep 2015.

[27] K. Barlee. (2017, Oct). FS-FBMC Based Communication in the FM Radio Band for Smart City Applications: Demodulated FM Signals. [Online]. Available: http://dx.doi.org/10.15129/ 487f6398-710d-4529-8233-48aa07acc3c7.

[28] Z. Zhao, N Vucic, M. Schellmann, "A simplified scattered pilot for FBMC/OQAM in highly frequency selective channels", in ISWCS'14, Barcelona, ES, pp. 819-823, Aug 2014. 